\title{
Investigation of a Control Function Determining the Location of the Zeros as a Possible Proof of the Riemann Hypothesis.
}

\author{
Miroslav Sukenik and Magdalena Sukenikova \\ Slovak University of Technology, Radlinského 9, 81237 \\ Bratislava. Slovakia \\ (sukenik4@gmail.com)
}

\begin{abstract}
The article examines the control function in relation to the distribution of Zeros on the critical line $x=0,5$. To confirm this hypothesis, it will be necessary to perform a large number of statistical analyzes of the distribution of non-trivial zero points of the Riemann Zeta function.
\end{abstract}

\section{1: INTRODUCTION}

The Riemann hypothesis was first formulated by the German mathematician Bernhardt Riemann in 1859. It reads: All nontrivial zero points of the Riemann Zeta-function have a real part equal to 1/2. Numbers whose real part is equal to $1 / 2$ form a line in the complex plane, which is called the critical line.

Proving the Riemann hypothesis would solve a large number of deep problems in various areas of mathematics (especially number theory), not only because in 2000 it was included among the 7 most important unsolved mathematical problems of the new millennium (so-called millennium problems). The Riemann Zeta function is defined as the sum of an infinite series.

$\zeta(s)=1+\frac{1}{2^{s}}+\frac{1}{3^{s}}+\frac{1}{4^{s}}+\ldots=\sum_{n=1}^{\infty} \frac{1}{n^{s}}$

Euler discovered the following relationship between the Zeta function and primes:

$$
\zeta(s)=\sum_{n \geq 1} \frac{1}{n^{s}}=\prod_{p \in P} \frac{1}{1-p^{-s}},
$$

A large number of scientific and popular articles, books, reflections, essays, etc. have been written about this hypothesis. One of the best (if not the best) is the British mathematician J Derbyshire's book "Prime Obsession", published in 2003 [1]. The book contains the history of prime numbers as well as the history of the Riemann hypothesis and their relation to quantum mechanics and cryptography.

One way to approach this issue is to investigate of control functions that could affect the distribution of non-trivial zeros. 


\section{2: Zeros Deployment Control Function.}

In 1859, Riemann proved the relation in which the Zeta function and the Euler Gamma function appear.

$$
\pi^{-s / 2} \Gamma\left(\frac{s}{2}\right) \zeta(s)=\pi^{-(1-s) / 2} \Gamma\left(\frac{1-s}{2}\right) \zeta(1-s)
$$

The following applies to the hypersphere area with dimension $\mathrm{s}=\mathrm{n}$ :

$S_{n}=\frac{2 \pi^{n / 2}}{\Gamma\left(\frac{1}{2} n\right)}$.

From (3) and (4) we get an interesting relationship between the Zeta function and the area.

$$
\frac{\zeta(s)}{\zeta(1-s)}=\frac{S(s)}{S(1-s)}
$$

By generalizing relation (5) we get the function:

$y=1 /(1-x)$

It is clear that relations (3) - (6) have no solution for $\mathrm{s}=\mathrm{x}=1$ because

$\zeta(1)=1+\frac{1}{2}+\frac{1}{3}+\cdots=\infty$

and Gamma(0) is undefined.

To highlight the resolution, we use a modified of function (6):

$y=\frac{1}{1-x^{2}}$

Here is its graph artificially stretched along the x-axis:

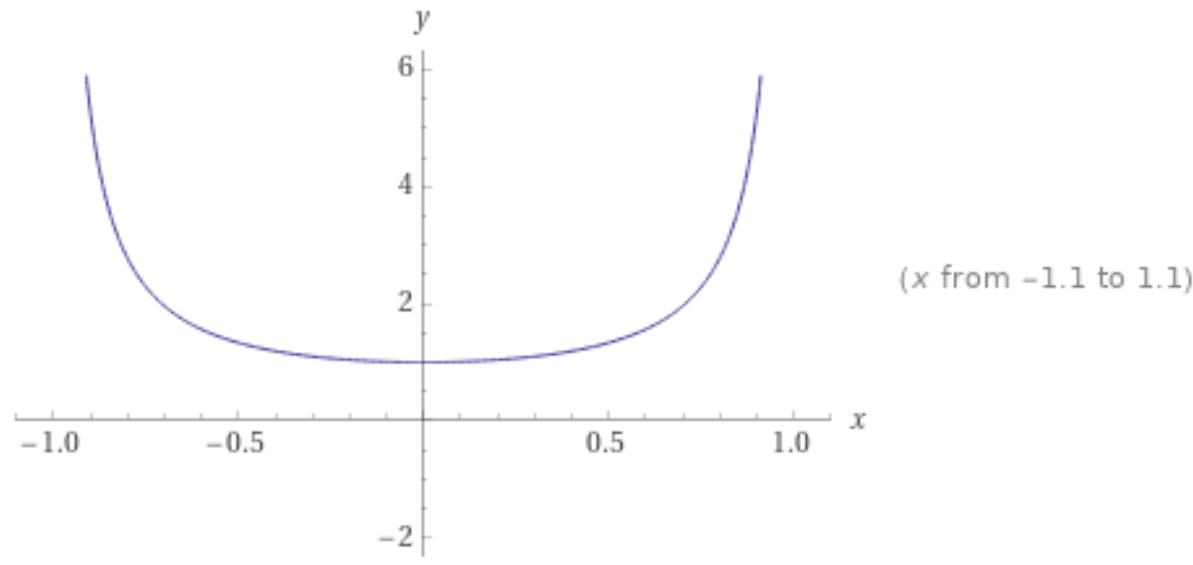


We are only interested in the values of $y$ for $0<x<1$ We assume that the values of function (7) will be projected on the line $x=0.5$. Therefore, especially in the interval $x$ ( 0 to 0.5 ) we find more Zeros (Z) than assumed by relation (8)

$Z=\frac{N}{2 \pi} \log \left(\frac{N}{2 \pi}-1\right)$

The projection of the function (7) on the line $\mathrm{x}=0.5$ can be expressed as follows:

$F(x)=F(a)=\frac{1}{y(x)-1} \int_{0}^{a} \sqrt{1+\left(\frac{\partial f(x)}{\partial x}\right)^{2}} d x$

As $\mathrm{f}(\mathrm{x})$ we used function (7). The factor $\mathrm{F}(\mathrm{x})$ indicates how much the corresponding length of the curve is greater than the value of $y$ at point $x$. Thus, how much more can we find zero points in a given interval than assumption (8).

First 100000 Zeros Z (r) were found in an interval of approximately 74000 (N). From relation (8) it follows that the number of $\mathrm{Z}(\mathrm{p})$ is 36000 . The correction $\mathrm{F}(\mathrm{x})$ is approximately 2.77 and according to relation (9) the answer $\mathrm{x}=0.35$.

Another measurement was made by Oldyzko [2], $\mathrm{N}=4.26$ 10exp7, Z (p) = $4.10 \exp 7, \mathrm{Z}(\mathrm{r})=$ 10exp8, Correction $\mathrm{F}(\mathrm{x})=2.5$ approximately $\mathrm{x}=0.38$. These two measurements are important for the calibration of our curve (7). For completeness only, we present other calculated corrections:

$\mathrm{F}(0.3)=3.247$

$\mathrm{F}(0.33)=2.938$

$\mathrm{F}(0.34)=2.848$

$\mathrm{F}(0.35)=2.77$

$\mathrm{F}(0.37)=2.605$

$\mathrm{F}(0.38)=2.53$

$\mathrm{F}(0.4)=2.3995$

$\mathrm{F}(0.5)=1.895$

$\mathrm{F}(0.6)=1.568$

$\mathrm{F}(0.7)=1.342$

Approximately from $\mathrm{x}=0.5$ an ever smaller decrease in $\mathrm{F}$ (x) begins and will approach indefinitely to 1 . The length of the curve up to the point $x=0.5$ is equal to $0.631 \ldots \ldots$ This is close to the value of the Golomb - Dickman constant, which has the value $0.642 \ldots$., This constant can be expressed as follows:

$$
\int_{0}^{\infty} \frac{f(x)}{x_{\text {Para } x>2}^{2}} d x=\int_{0}^{1} e^{\operatorname{Li}(n)} d n
$$

We see that the function $\mathrm{Li}(\mathrm{n})$ appears in this integral, which is related to prime numbers and thus also to Riemann's Zeta function. More about the distribution of zero elements vid. [3], [4]. 


\section{3: Conclusions:}

If the changes in the factor $\mathrm{F}(\mathrm{x})$ were confirmed as much as possible, it would be a sign that Zeros really lie on the critical line $\mathrm{x}=0.5$.

It would be appropriate to independently research and statistically process multiple control functions, e.g. (6) and similar function types. It is interesting that in the case of function (6) the length of the curve is from the interval $\mathrm{x}=0$ to $\mathrm{x}=0.5$ is $\mathrm{D}=\mathrm{F}(0.5)=1.132 \ldots$ which corresponds to the Viswanaths constant.

$$
\lim _{n \rightarrow \infty}\left|a_{n}\right|^{\frac{1}{n}}
$$

where a (n) is the Fibonacci sequence.

NOTE: Complex associated numbers are displayed by projecting the negative value of the function (7) on the critical line $\times 0.5$.

\section{References:}

[1]: Derbyshire,J. „Prime Obsession: Bernhard Riemann and the Greatest Unsolved Problem in Mathematics“ ,Joseph Henry Press, (2003)

[2]: Hiary,A,G,, Oldyzko,A. „THE ZETA FUNCTION ON THE CRITICAL LINE“ MATHEMATICS OF COMPUTATION“, Volume 81, Number 279, (July 2012)

[3]: Benatar,J,,Borichex,A,,,Sodin,M. "Zero distribution of power series and binary correlation of coefficients", arXiv:2104.04812, (2021)

[4] : Chavez,D,, Allawala,A. "Prime zeta function statistics and Riemann zero-difference repulsion“, arXiv:2102.02280, (2021) 